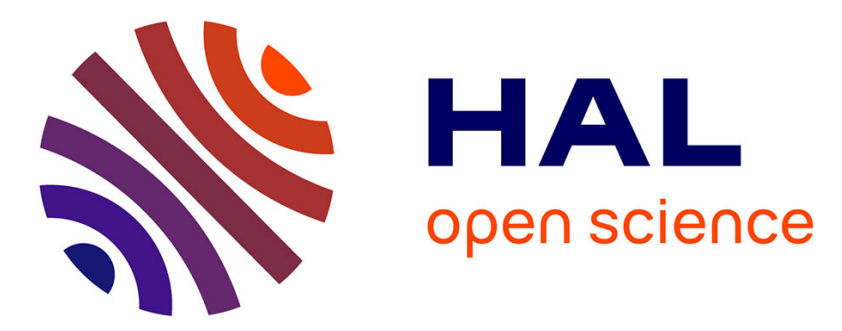

\title{
PARTICLE EMISSION BUT ALSO FISSION IN THE DECAY OF VERY EXCITED NUCLEI
}

\author{
H. Delagrange, C. Gregoire, Y. Abe, N. Carjan
}

\section{To cite this version:}

H. Delagrange, C. Gregoire, Y. Abe, N. Carjan. PARTICLE EMISSION BUT ALSO FISSION IN THE DECAY OF VERY EXCITED NUCLEI. International Conference on Heavy Ion Nuclear Collisions in the Fermi Energy Domain Hicofed 86, 1986, Caen, France. pp.C4-305-C4-315, 10.1051/jphyscol:1986434 . jpa-00225800

\section{HAL Id: jpa-00225800 https://hal.science/jpa-00225800}

Submitted on 1 Jan 1986

HAL is a multi-disciplinary open access archive for the deposit and dissemination of scientific research documents, whether they are published or not. The documents may come from teaching and research institutions in France or abroad, or from public or private research centers.
L'archive ouverte pluridisciplinaire HAL, est destinée au dépôt et à la diffusion de documents scientifiques de niveau recherche, publiés ou non, émanant des établissements d'enseignement et de recherche français ou étrangers, des laboratoires publics ou privés. 
JOURNAL DE PHYSIQUE

Colloque C4, supplément au $n^{\circ} 8$, Tome 47, août 1986

PARTICLE EMISSION BUT ALSO FISSION IN THE DECAY OF VERY EXCITED NUCLEI

H. Delagrange, C. GREgoire, Y. ABE* and N. CARJAN**

GANIL, BP 5027, F-14021 Caen Cedex, France

*RIFP, Kyoto University, Kyoto 606, Japan

* CENBG. Le Haut Vigneau, F-33170 Gradignan, France

Résumé - Afin d'analyser le comportement des noyaux très excités, nous présentons une extension du modèle statistique avec une dépendance explicite du temps. Les dépendances de la barrière de fission en fonction de la température et du moment angulaire sont obtenues dans le cadre de la goutte liquide. Ce cadre permet de définir les paysages de potentiel associēs aux quelques variables collectives qui évoluent, pendant la décroissance, de la manière décrite par une équation de transport général isée. Nous calculons l'évaporation de particules légères durant le processus de fission rapide et également en compétition avec la fission suivant la formation d'un noyau composé. Ces calculs sont comparés avec quelques résultats expérimentaux, notamment ceux de ${ }^{40} \mathrm{Ar}+238 \mathrm{U}$ à $27 \mathrm{MeV} / \mathrm{u}$. Le refroidissement s'établissant pendant le transfert d'énergie, par l'évaporation de particules, empêche la répartition dans le système composite de toute l'énergie disponible. Ces noyaux doivent être plutôt qualifiés de tièdes que de vraiment chauds.

\footnotetext{
Abstract - An extension of the statistical model for the study of very excited nuclei is presented. The temperature and angular momentum dependences of the fission barriers are obtained in a liquid drop picture. It provides us with the relevant potential landscapes for some collective variables which evolve during the decay according to a generalized transport equation. The light particle evaporation during the fast fission process and the fission after compound nucleus formation is calculated and compared with some available experimental results, e.g. for ${ }^{40} \mathrm{Ar}+238 \mathrm{U}$ at $27 \mathrm{MeV} / \mathrm{u}$. The competing process of particle emission (cooling) during the thermal equilibrium prevents a complete transfer of excitation energy along the whole evolution of the system. Such nuclei should be called warm instead of hot.
}

\section{I - INTRODUCTION}

The heavy ion reactions in the intemediate energy open a new field of investigation for the physical properties of nuclei at high temperature (or high excitation energy). Several regimes can be expected for the decay of an highly excited nucleus :

i) For a temperature domain below $2 \mathrm{MeV}$, the standard statistical model applies, since the equilibration time $\tau$ for the internal degrees of freedom is much smalier than the typical time for fission decay $\left(\tau_{f}\right)$ and for particle evaporation $\left(\tau_{p}>\tau_{f}\right) / 1 \%$.

iif For a temperature range between 2 and $5 \mathrm{MeV}$, a dynamical competition between 
fission and particle decay is expected to be crucial, since $\tau_{p}$ becomes much smaller than the transient time for fission (which is a fraction of $\tau_{f}$ ). In this situation, the temperature and the angular momentum dependence of the fission barrier plays an important rôle in the dynamical evolution and could be seen in the particle multiplicity associated to fission $/ 2 \%$.

iii) At excitation energies above 5-6 MeV per nucleon, a cracking or multifragmentation is predicted during the expansion of the system, at the freezeout density and for a thermodynamical equilibrium $/ 3 /$. In dynamical estimates, at an higher excitation energy with an associated temperature close to the critical temperature, a vaporization is very likely above a flash temperature $14 /$.

In nucleus-nucleus collisions with a bombarding energy between 20 and $100 \mathrm{MeV} / \mathrm{u}$, an entrance channel limitation to the deposition of energy could hinder to reach the third regime. Furthermore, detailed balance calculation /1/ and hot Hartree-Fock calculation $15 /$ indicate that $\tau_{7}$ is as short as $10^{-22} \mathrm{~s}$ for a temperature of $T=5 \mathrm{MeV}$ (in the case of the ${ }^{2} \mathrm{P} 8 \mathrm{~Pb}$ nucleus). Consequently, during the damping of the relative motion which converts the available kinetic energy into excitation energy, one couTd imagine that the cooling of the system starts before the total energy deposition.

A particular decay channel is the fission after compound nucleus formation or, in absence of fission barrier for the composite system, the fast fission process $/ 6 /$. The collective dynamical paths of both channels are surely modified by the particle evaporation. Similarly, the associated particle evaporation is certainly determined by the collective path and the variation of the collective potential landscape and the angular momentum. In this work, these contributions to the symmetric fragmentation component of the mass distributions have been considered in the case where $\tau_{e}<\tau_{p} \leqslant \tau_{f}$, i.e. in the second regime of deexcitation. A generalized transport equation is described in the section II, completed by a few static considerations about the variation of the liquid drop energies and of the fission barrier with the temperature and/or the angular monentum. The partial wave sharing between the fission, fast fission and evaporation residues cross sections is indicated by referring to a friction model for dinuclear systems. In the section III, realistic calculations concerning the statistical decay of hot heavy nuclei are reported for an example where a pure fast fission is expected. These calculations have been also performed for medium systems where the fission after compound nucleus formation can occur for the lowest partial waves. The results are given in the section IV, with a comparison to some available experimental data.

\section{II - THEORETICAL OUTLINE}

In the recent years, transport equations have been widely used to describe the evolution towards equilibration of systems formed in heavy ion induced reactions. The path towards equilibration is followed through dissipation of collective motion. An overview of these approaches can be found in $17 /$. Al though the transport treatment has been mainly applied to the deep-inelastic reactions, recent investigations have focused also on reactions without fission barrier (fast-fission mechanism) /6/ and even on the evolution of compound nuclei towards fission /2,8-11/ extending the pionneer work of Kramers $/ 12 /$. We will present in this paragraph the introduction of the particle emission in the different transport equations governing on one hand the fast-fission mechanism and on the other hand the evolution of compound nuclei.

II.1- Dynamical competition between particle emission and fission decay after compound nucleus formation

A generalization of the Fokker-Planck equation governing the path to fission has been proposed in $/ 2 /$ in order to take into account the particle emission. The distribution d $\left(Q, P, t ;\left\{s_{j}\right\}\right)$ fulfills the following equation : 
$\frac{\partial}{\partial t} d(Q, P, t ;\{s\})=-\frac{P}{B} \frac{\partial d}{\partial Q}+\frac{\partial U}{\partial Q} \frac{\partial d}{\partial P}+\frac{\gamma}{B} \frac{\partial}{\partial P}(P d)+\gamma^{T} \frac{\partial^{2}}{\partial P^{2}} d-\sum_{i=1}^{k} \frac{\partial}{\partial s_{i}}\left(\Gamma_{i} d\right)$

with $Q$ the elongation coordinate; $P$, its conjugate momentum ; $B$ and $\gamma$ the inertia and the viscosity associated to the dynamical motion along the $Q$ coordinate; $U$, the potential, $T$ the temperature $;\left\{s_{j}\right\}$ the coordinate corresponding to particle emission ( $i, e,, n, p, \alpha)$ and $r_{i}$ the decay width for particle of type $i$. The variables $B$ and $\gamma$ will be described in more details in the paragraph (1I.3). The decay widths are calculated with the help of the detailed balance principle $/ 2 /$.

The generalized transport equation Eq.1 is solved numerically with the help of the technique of propagation with gaussian bundles $/ 13,14 /$. The inital condition for the distribution function $\mathrm{d}\left(\mathrm{Q}, \mathrm{P}, \mathrm{t}=0 ;\left\{\mathrm{s}_{\mathbf{i}}\right\}\right)$ reads :

$$
d\left(Q, P, t=0 ;\left\{s_{i}\right\}\right)=\delta\left(Q-Q_{\min }\right) \cdot \frac{1}{\sqrt{2 \pi B T_{i}}} \exp \left(-\frac{P 2}{2 B T_{i}}\right) \delta\left(\left\{s_{j}\right\}\right)
$$

where $Q_{\min }$ is the elongation coordinate at ground-state deformation and $T_{i}$, the initial temperature of the compound nucleus. The excitation energies dealt with in the heavy-ion induced reactions we will investigate are rather large and therefore a gaussian distribution in momentum corresponds to a rational choice since the thermal fluctuations are prominent. In this theoretical approach, the overall fission probability is given for a given angular momentum by :

$$
P_{f}=\int_{0}^{\tau} P_{f}(t) \cdot \bar{f}\left(t ; s_{i}^{0}(t)\right) d t
$$

where $\bar{f}\left(t ; s_{j}\right)$ is the probability to find an excited nucleus with a charge and a mass number $(z, A)$ for mean numbers $s_{j}$ of emitted particles. It is equal to the integral of the density function $d_{j}$ performed over $P$ from $-\infty$ to to and over $Q$ from $-\infty$ to $Q_{S}$ (location of the saddle point). The overall probability $P_{E R}$ of evaporation residues is then deduced from $P_{f}$ :

$$
P_{E R}=1-P_{f}
$$

The average particle multiplicities deduced from these calculations are of three types : i) particle multiplicity associated to the fission fragments

$$
<v>=\frac{\int_{i F}^{\tau} P\left(t, s_{i}^{0}(t)\right) s_{i}^{0}(t) d t}{\int_{0}^{\tau} P_{f}\left(t, s_{i}^{0}(t)\right) d t}
$$

where $P_{f}$ is the fission probability and $\tau$ the total decay time.

The multiplicity associated to fission fragments before scission is given by :

$$
\left\langle v_{i}\right\rangle F S=\frac{\int_{0}^{\tau} P_{F}\left(t, s_{i}^{0}(t)\right)\left[s_{i}^{0}(t) \zeta(t)\right] d t}{\int_{0}^{\tau} P_{f}\left(t, s_{i}^{0}(t)\right) d t}
$$

where $\zeta(t)$ is the probability to emit the considered particle before reaching the scission point. It is equal to 1 up to the average time spent to go from the saddle to the scission point; it is equal to zero otherwise.

ii) The multiplicity associated to the compound nucleus (evaporation residues and 
fission fragments as well) before reaching the saddle point :

$$
\left\langle v_{i}\right\rangle=\frac{\int_{0}^{\tau-} f\left(t ; s_{i}^{0}(t)\right) s_{i}^{0}(t) d t}{\int_{0}^{\tau-} f\left(t ; s_{i}^{0}(t)\right) d t}
$$

The multiplicity associated to the evaporation residues is also given by eq. 6 when $f(t ; s)(t))$ is replaced by $f_{E R}(t ; s P(t))$. This last quantity is the probability to find an excited nucleus with a charge ( $Z$ ) and a mass number (A) not decaying subsequently: by fission.

iii) The "statistical model" multiplicity associated to fission fragments :

$$
\left\langle v_{j}\right\rangle S M=\frac{\int_{0}^{\tau} p_{f}^{K r a m e r s} s_{i}^{o}(t) d t}{\int_{0}^{\tau} p_{f}^{K r a m e r s} d t}
$$

where $P_{f}^{K r a m e r s}$ is the "Kramers" fission probability. It is statistical, in the sense that $P$ kramers is not depending on time explicitely but it is slightly different from the common usage in statistical decay codes because the fission barrier is temperature dependent.

The statistical model multiplicity of particles emitted before scission is obtained following the procedure presented for the case $i), i$.e. Weighting $s_{i}^{0}(t)$ by $\zeta(t)$ in the Eq. 7.

\section{I1.2 - Fast fission and particle emission}

The transport equations dealing with fast-fission incorporate dissipation along the collective coordinates $\left(R, \theta, x, y,\left\{s_{j}\right\}\right)$. The variable $R$ is the radial distance between the two partners. The angle defined by the axe going through their centers of mass and the beam axis is $\theta$. The coordinates $x$ and $y$ are the mean asymmetry of the system i.e. $x=\left(A_{2}-A_{1}\right) /\left(A_{2}+A_{1}\right)$ and the neutron excess $y=N_{1}-z_{1}$. The atomic mass number and neutron number are denoted by $A, N$ and $Z$ respectively; the subscripts 1 and 2 are the corresponding indexes for the light and the heavy fragments. The ensemble of the emitted particles $(i=n, p, \alpha)$ is $\left\{s_{j}\right\}$. To the equations of motion describing the evolution of the system in the $(R, \theta, x, y)$ space $/ 6 /$, we add a depletion term due to particle emission defined as :

$$
\frac{d s_{i}}{d t}=\Gamma_{i}\left(\Delta E^{*}\right) / \hbar
$$

where $\Delta E^{\star}$ is the thermal excitation energy gained from dissipation in the other collective degrees of freedom. The set of the coupled differential equations including Eq. 7 is solved with the help of a corrector-predictor method.

\section{II.3 - Potential landscape}

The potential landscape as functions of the various collective variables governing the dynamical behaviour of the reaction is determined as a time-dependent linear combination of a sudden and a liquid drop potential /6/ with a centrifugal correction added. The liquid drop potential (for all shape configurations) includes 
a temperature dependence defined from the theoretical investigation of $/ 15,16 /$. The parametrization of $/ 17 /$ with an effective interaction of the Bruckner type provides a rather good analytical formula for the symmetrical fission barrier.

For various couples of temperature and angular momentum, the symmetrical fission barrier vanishes as displayed in Fig. 1 for the $185 \mathrm{Ir}$. In this figure, the potential was calculated for the parametrization of $/ 18 /$ and the temperature variation of the liquid drop expressions from $/ 15 /$. The centrifugal tem is obtained with moment of inertia for diffuse surfaces (full line) or for rigid body (dotted line). In the first case, the diffuseness follows the same temperature evolution as for the coulomb potential.

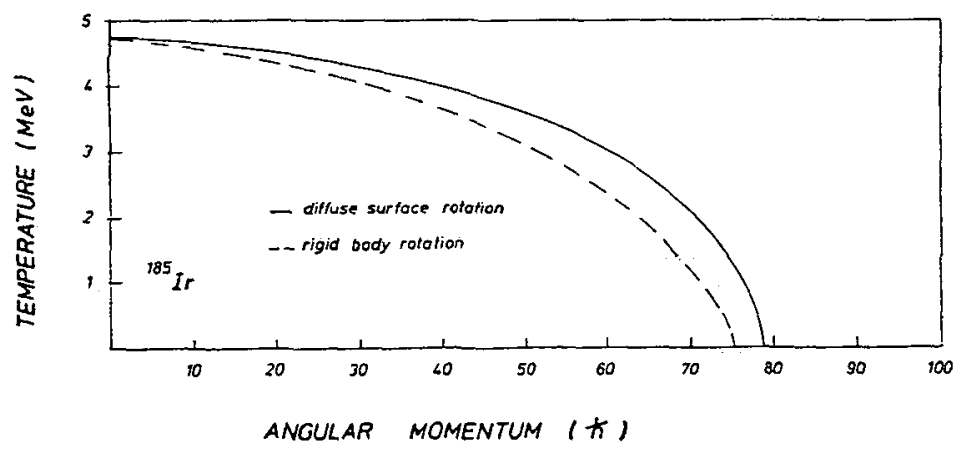

Figure 1 : Evolution of the temperature corresponding to the vanishing of the fission barrier $185 \mathrm{Ir}$ as a function of angular momentum. The dashed curve is obtained with a centrifugal contribution considering a rigid body moment of inertia. The solid curve concerned calculations including a correction to the rigid monent of inertia due to a temperature dependent surface diffuseness.

The limit temperature at which the symmetrical fission barrier vanishes is a key parameter to precise the dynanics of the reaction. In the case of $40 \mathrm{Ar}+238 \mathrm{U} / 19 /$, the fission barrier is almost completely nonexistent and fast-fission is called for. In the case of systems leading to $185 \mathrm{Ir} / 20 /$ at an excitation energy of $164 \mathrm{MeV}$ corresponding to a temperature of $2.75 \mathrm{MeV}$ (if all the available energy were dissipated in thermal energy), over $1=55-63$ (see fig. 1), there is no fission barrier. We face then a mixture of equilibrated fission (fission after compound nucleus formation) and fast-fission. In such a situation where the barrier is temperature dependent and cooling of the system by particle evaporation, is present, a compound nucleus formation can result for a reaction starting with an initial fast-fission evolution. This interplay between these two components is of prime interest in order to analyse the experimental data for a wide range of heavy ion induced reactions, and deduce the conditions of collective dissipation.

\section{III- STATISTICAL DEEXCITATION OF HOT HEAVY SYSTEMS}

We shall consider in this section the deexcitation of systems mainly during the entrance channel of a heavy-ion collision. A way to analyse this deexcitation process as a function of the entrance channel is to select a reaction mechanism where the compound nucleus formation is very unlikely. The fast fission phenomenon provides a fragment distribution close to the fission fragment distribution observed after compound nucleus formation. In fact, the angular momentum 1 and the excitation energy $E^{*}$ cancel both the fission barrier, if any. For very heavy systems $/ 6 /$, a small amount of $\left(1, E^{*}\right)$ is sufficient in order to make the fission barrier 
vanishingly small; consequently, pure fast fission js expected for these systems. Since the 1 ight particle evaporation reduces 1 and $E^{*}$, it is of particular relevance to study it during the dynamical path.

In the intermediate energy domain, the momentum transfer distritution in central collisions ranges from very incomplete transfer to the complete transfer, with a smaller probability in this later case $/ 21,22 \%$. We shall report on numerical calculations performed according to the theoretical treatment described in the section II. First, a complete linear momentum transfer is simulated by a friction model for the ${ }^{40 \mathrm{Ar}}(27 \mathrm{MeV} / \mathrm{U})+238 \mathrm{U}$ system. Secondly, the $28 \mathrm{Si}(27 \mathrm{MeV} / \mathrm{u})+238 \mathrm{U}$ system is considered in order to mock up an incomplete momentum transfer corresponding to the experimental mean value for the momentum transfer distribution in the $40 \mathrm{Ar}(27 \mathrm{MeV} / \mathrm{U})+238 \mathrm{U}$ reaction. A rather central collision is chosen in both cases with a partial wave $1=5$. The friction model with associated friction and inertial coefficients to the collective coordinates can be found in $/ 6 /$.

\section{III.1 - Complete linear momentum transfer}

In the case of the ${ }^{40} \mathrm{Ar}(27 \mathrm{MeV} / \mathrm{U})+238 \mathrm{U}(1=5)$ system, a typical fast fission trajectory is found, because no fission barrier can contain the system in a compact configuration. The mass asymnetry relaxes partly before the reseparation, which occurs after a reaction time of $610^{-21}$ s. During the first stage of the collision, the friction forces convert the main part of the available kinetic energy into excitation energy, as it can be seen in the figure 2 . The temperature reaches

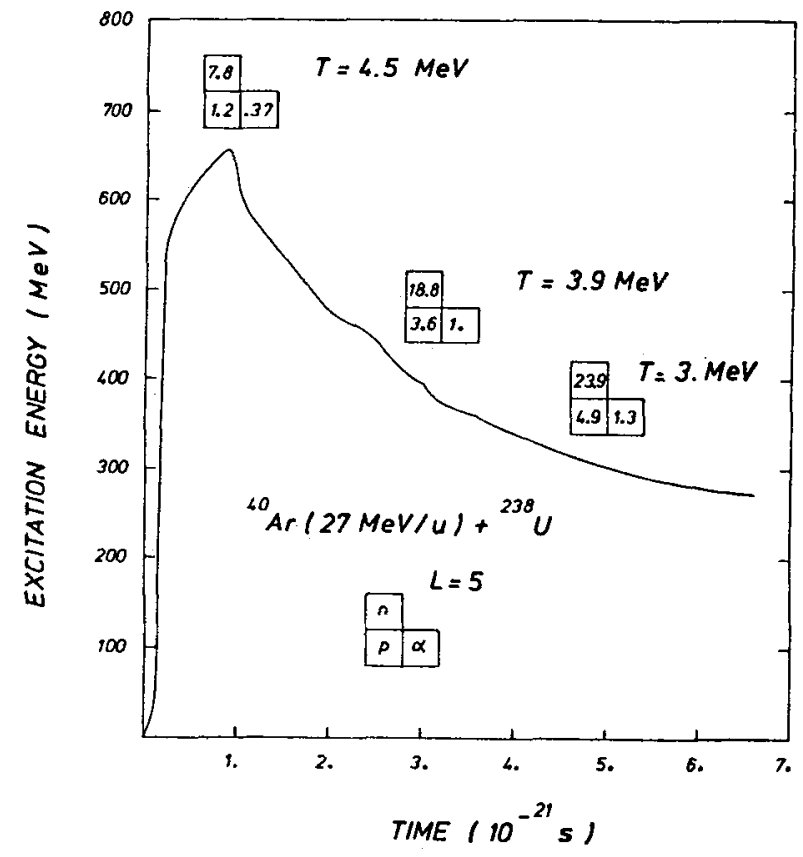

Figure 2 : Time variation of excitation energy, temperature $T$, and particle multiplicities for the fast-fission of $40 \mathrm{Ar}$ $(27 \mathrm{MeV} / \mathrm{u})+238 \mathrm{U}$ at $1=5$.

4.6 MeV. From the previous considerations, light particle emission is expected to occur very quickly. This particle emission is calculated with its coupling to the dynamical evolution of the collective variables, according to equation 8 . It produces a substantial cooling of the system, during its stay in the entrance channel potential pocket and before the decay inside the fission valley. This epoch is long enough for a decrease by $350 \mathrm{MeV}$ of excitation energy. At the reseparation 
the mean numbers of neutron, proton and alpha emitted particles are 25.6, 5.32, 1.43, respectively. In experimental analysis these emitted particles are associated to the fission-like products arising from the fast fission process.

\section{III.2 - Incomplete linear momentum transfer}

The dynamical evolution of the ${ }^{28} \mathrm{Si}(27 \mathrm{MeV} / \mathrm{u})+238 \mathrm{U}$ system at $1=5$ in the radial distance-mass asymmetry two dimensional display is given in figure 3 . The mean numbers of neutron, proton and alpha emitted particles along the collective dynamical path are given for each $10^{-21} \mathrm{~s}$. One can observe the relaxation in mass asymetry up to $x=.15$. The system lies in a compact configuration for almost $10^{-20} \mathrm{~s}$ with a radial distance between the emerging fragments between $10 \mathrm{fm}$ and 12 $\mathrm{fm}$. The absence of fission barrier for the total system allows the reseparation and the so-called fast fission. The large amount of emitted particles before scission is due to the large time scale, as compared to the mean emission time at the involved excitation energy (the maximum value is $504 \mathrm{MeV}$ in this example). From this calculation, one expects consequently an entrance channel effect on the particle multiplicity, because the fast fission reaction time depends substantially on the considered initial system.

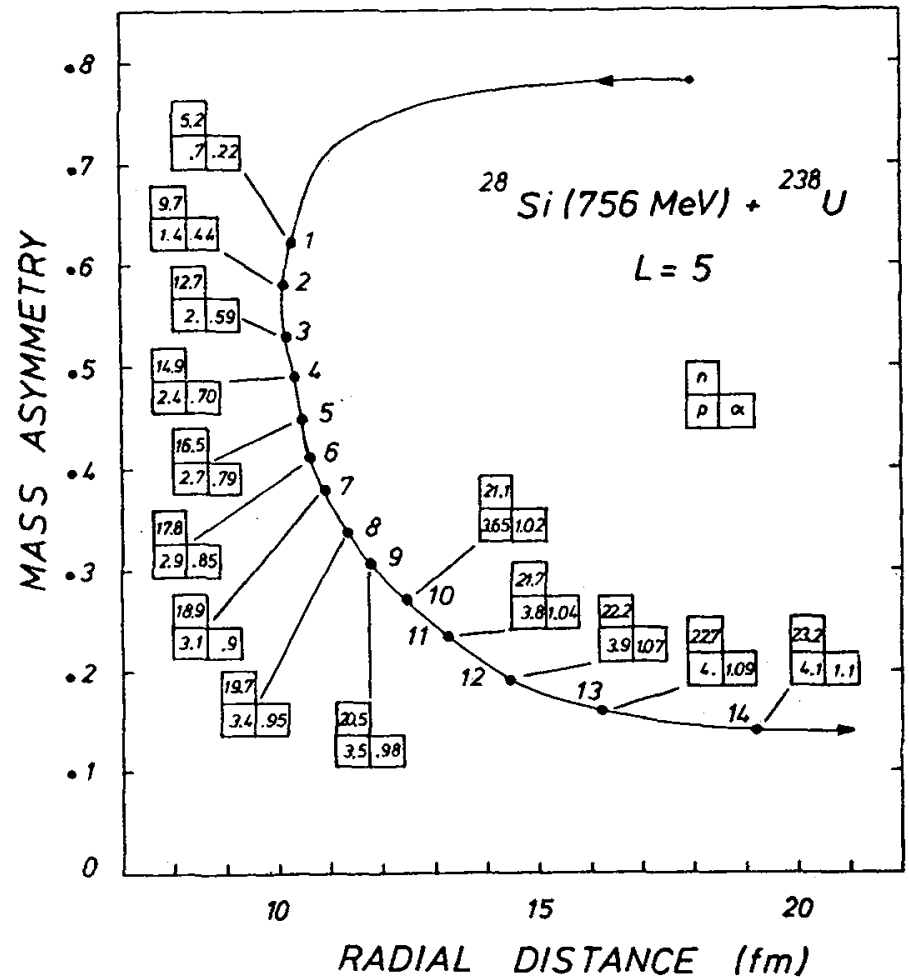

Figure 3 : Fast-fission trajectory in a radial distance-mass asymmetry map for the ${ }^{28} \mathrm{Si}$ $(27 \mathrm{MeV} / \mathrm{u})+238 \mathrm{U}$ system at $1=5$. At each $10^{-21} \mathrm{~s}$, the mean numbers of evaporated neutron, proton and alpha particles are indicated, up to the reseparation.

\section{III.3 - Temperature dependence of the fission barrier in pure fast fission cases}

In the two previous calculations, no temperature dependence of the fission barrier was taken into account. Since it could modify the associated particle multiplicity, it is worthwhile to compare calculations with and without introduction of the temperature dependence of the barrier /15/. The comparison is done in the table 1 for various partial waves. In both cases, the calculated values for charged particles are close to the data $/ 19,21 /$. 
The particle multiplicities decrease when the temperature correction for the fission potential landscape is done. As a matter of fact, this correction flattens the potential and washes out the shoulder along the radial distance coordinate found at zero temperature. The reseparation is consequently faster in those cases and the evaporation before scission is cut short. If the temperature dependence of the potential could modify the duration of the collisions with variation up to $20 \%$ of the particle multiplicity, it could also influence the precise location of the frontier between the fast fission and the compound nucleus formation. It is one of the topics of the next section.

\begin{tabular}{|c|c|c|c|c|c|c|c|c|}
\hline (K units) & $\begin{array}{c}E_{1}^{\text {loss }} \\
(\mathrm{MeV})\end{array}$ & $\begin{array}{c}E_{2}^{\text {Toss }} \\
(\mathrm{MeV})\end{array}$ & $\langle n\rangle_{1}$ & $\langle n\rangle_{2}$ & $\langle p\rangle_{1}$ & $\langle p\rangle_{2}$ & $\langle\alpha\rangle_{1}$ & $\langle\alpha\rangle_{2}$ \\
\hline 5 & 620 & 634 & 23.2 & 21.79 & 4.12 & 3.87 & 1.12 & 1.07 \\
\hline 55 & 613 & 629 & 21.26 & 20.31 & 3.69 & 3.54 & 1.03 & 0.99 \\
\hline 105 & 596 & 605 & 18.80 & 16.58 & 3.14 & 2.72 & 0.90 & 0.79 \\
\hline 155 & 572 & 586 & 15.05 & 12.82 & 2.21 & 1.96 & 0.69 & 0.66 \\
\hline
\end{tabular}

TABLE 1 Influence of the temperature correction of the fission valley on the particle multiplicities, for a pure fast fission process in the $28 \mathrm{Si}(756 \mathrm{MeV})+$ ${ }_{238} \mathrm{U}$ system. 1. Without temperature dependence. - 2. With temperature dependence.

IV - DYNAMICAL DECAY OF EXCITED NUCLEI FORMED DIRECTLY THROUGH COMPOUND NUCLEUS OR AFTER FAST FISSION : CASE OF $185 \mathrm{Ir}$.

Recently, Zank et a1. /20/ measured neutron evaporation correlated either to evaporation residues (ER) or fission fragments (FF). They studied the three following reactions: $40 \mathrm{Ar}+14 \mathrm{IPr} \rightarrow 18 \mathrm{Ir}, 20 \mathrm{Ne}+165 \mathrm{Ho} \rightarrow 185 \mathrm{Ir}$ and ${ }^{12} \mathrm{C}+175 \mathrm{Lu} \rightarrow$ 187 Ir with appropriate bombarding energies to reach the same excitation energy $E^{\star}=164 \mathrm{MeV}$. Apart from neutron emission multiplicities, they determined $\mathrm{ER}$ and FF cross-sections ; from them they deduced the fusion cross-sections (and the associated $1_{\mathrm{cr}}$ critical angular momenta for fusion) for the three considered systens. Even, if the cross-sections for ER and FF production are well reproduced with the help of the statistical model, the multiplicities of neutron emission before fission are incompatible with such an approach. This physical situation seems quite appropriate to be dealt with in our dynamical decay analysis.

In order to examine the contributions to the symmetric fragmentation component (fast fission + fission after compound nucleus formation) and the evaporation residue cross sections, a few limiting angular momentum values have to be defined, with the example of $40 \mathrm{Ar}+1^{41} \mathrm{Pr}$. Below $l_{\mathrm{gr}}(\sim 114)$, the critical angular momentum, fusion is expected. Between ${ }_{B f}=0(T=0$ MeV $)$ 80, the maximum value above which the fission barrier vanishes at zero temperature and $1_{c r}$, fast fission might occur. Between $1_{B f=0}\left(T=T_{m a x}\right) 55$ and $1_{B f=0}(T=0 M e V)$, dynamics will decide between a compound nucleus formation ( $C N F)$ and fast fission. Between 0 and $l_{B f=g}\left(T=T_{\text {max }}\right)$ CNF is expected. The low partial wave range will be considered firstly and the fast fission component secondly.

\section{IV.1 - Aspects due to compound nucleus deexcitation}

The compound nucleus formation is only possible if the initial composite nucleus (projectile + target) is hold together by a fission barrier. As presented in 
section II, such a system at an initial available excitation energy of $164 \mathrm{MeV}$ $C T=2.75 \mathrm{MeV}$, following the relationship, $E^{*}=2 T^{2}$ with $a$, the level density parameter taken from $/ 23 /$ ), has a fission barrier vanishing for 1 between 50 and 60 . Although the three composite systems are slightly different (181Ir, $185 \mathrm{Ir}, 187 \mathrm{Ir}$ ), 185 Ir has been considered in our calculations as representative of the three cases. The calculations of the dynamical particle decay of the compound nucleus on its way to fission yield the fission probability displayed in Fig.4. For value of 1 larger than 60 up to the various measured 1 crit, the fission probability is set equal to 1 even if fission does not occur there after complete equilibration of all degrees of freedom. The calculated cross-sections (ER and FF) deduced from this fission probability are in good agreement with the experimental data (see Table 2).

\begin{tabular}{|c|c|c|c|c|c|}
\hline \multirow[t]{2}{*}{ System } & \multirow{2}{*}{$\begin{array}{l}\mathrm{E}_{1 \mathrm{ab}} \\
(\mathrm{MeV})\end{array}$} & \multicolumn{2}{|c|}{$\sigma_{E R}(m b)$} & \multicolumn{2}{|c|}{$\sigma_{F F}(m b)$} \\
\hline & & $\exp$ & calc. & $\exp$ & calc. \\
\hline $12 \mathrm{C}+175 \mathrm{Lu}+187 \mathrm{Ir}$ & 192 & $1160 \pm 260$ & 1025 & $663 \pm 70$ & 807 \\
\hline $20 \mathrm{Ne}+165 \mathrm{Ho}+185 \mathrm{Ir}$ & 220 & $620 \pm 60$ & 590 & $1070 \pm 110$ & 1103 \\
\hline $40 \mathrm{Ar}+141 \mathrm{Pr} \rightarrow 181 \mathrm{Ir}$ & 316 & $220 \pm 50$ & 269 & $811 \pm 30$ & 766 \\
\hline
\end{tabular}

Table 2 : Theoretical and experimental cross-sections for production of evaporation residues (ER) and fission fragments (FF). Experimental data are taken from $120 /$. These calculated results refer only to true compound nucleus decay.

If only dynamical compound nucleus calculations are considered, the average neutron multiplicity associated to ERs reaches 10.5 neutrons for all three systems (see table 3). This value is closed to the experimental data. The calculated mean neutron multiplicity. for prefission emission of 2.8 neutrons stands siightly below the measured results (See table 3 ). Nevertheless, such a calculated multiplicity is larger than the similar quantity computed in the standard statistical model $/ 20 /$ at least for both systems ${ }^{40} \mathrm{Ar}+141 \mathrm{Pr}(\langle n\rangle=1.1)$ and $20 \mathrm{Ne}+165 \mathrm{Ho}\left(\left\langle v_{n}\right\rangle=1.9\right)$.

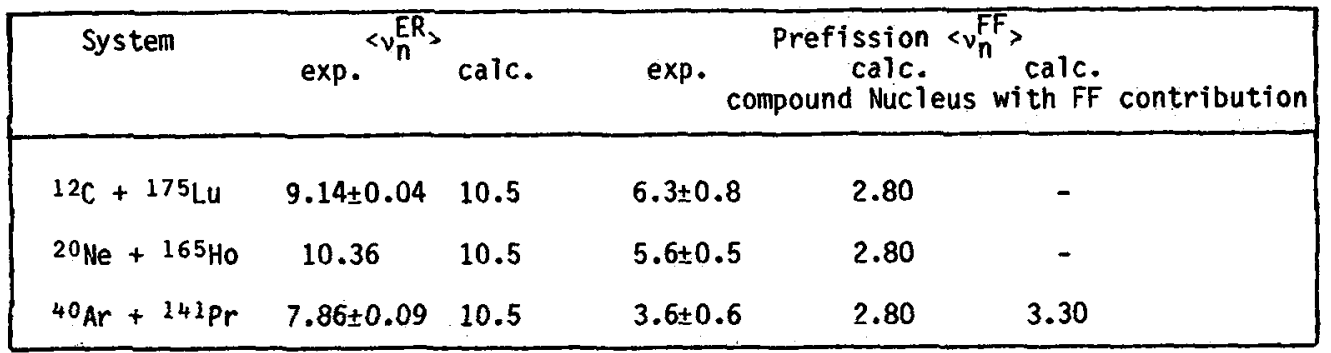

Table 3 : Experimental $/ 20 /$ and calculated neutron multiplicities associated with evaporation residues and fission before scission.

Existing discrepancies could be linked either to contributions arising from neutron emission taken place at angular momenta for which no fission barrier exists or because a proper consideration of incomplete fusion is called for (notably in the case of $12 \mathrm{C}+175 \mathrm{Lu})$. The latter cause of difference is yet not investigated in this study. The former cause will be dealt with in the next paragraph. 


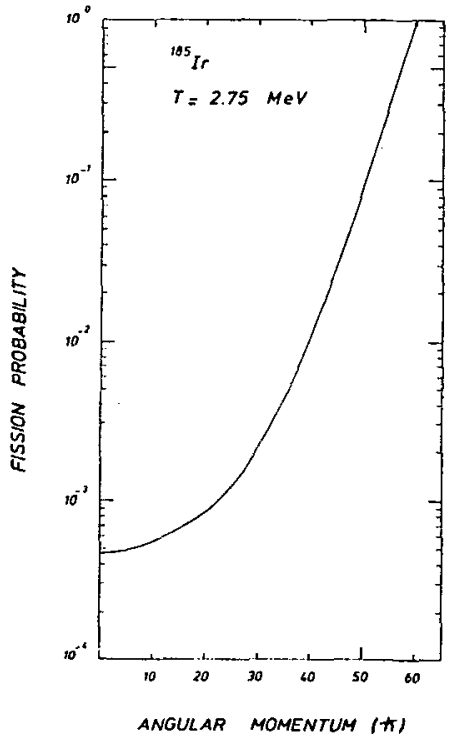

Figure 4 : Overall fission probability in the decay of $185 \mathrm{Ir}$ as a function of angular momentum. The initial temperature is $2.75 \mathrm{MeV}$. This probability results from calculations in the context of compound nucleus deexcitation.

\section{IV.2 - Aspects dure to fast-fission}

At least for the ${ }^{40} \mathrm{Ar}+141 \mathrm{Pr}$ case, in the 1 region between $\mathrm{I}_{\mathrm{B}} \mathrm{B}=0(\mathrm{~T}=0)$ and ${ }^{1} \mathrm{Cr}$, the system evolves in its potential 1 andscape on fast-fission trajectories. Th
evolution is similar to the case $40 \mathrm{Ar}+238 \mathrm{U}$ al ready presented in section III. Starting from the critical contact between the projectile and the target, the system will get excited and therefore emit light particles. Depending on excitation energy and angular momentum, two regimes stand up. On one hand, for the highest partial waves $(85<1<114)$, the system will emit a certain amount of particles (for $1=100$, $v_{n}=2.8$ ) before separating in a pure fast-fission mode. On the other hand, for the lower 1 range $\left(1_{B f=0}(T=2.75)<1<85\right)$, the system will spend more time along the corresponding fast fission trajectory and emit more particles (for $1=70, v_{n}=$ 4.3). In this situation, the particle emission cools down the system with regard to excitation energy and spin, to such an extent that a fission barrier builds up. Consequentiy, the system reaches the compound nucleus formation, but with a small excitation energy inhibiting the particle emission from the saddle to the scission point. The subsequent compound nucleus decay is then followed with the appropriate dynamical treatment for evaporation residue production (see section IV-A). The cross-section oER reaches $406 \mathrm{mb}$, i.e. a two-fold enhancement compared to true compound nucleus decay. This enhancement destroys the agreement with experimental data but clearly calculations considering ${ }^{181} \mathrm{Ir}$ and not only ${ }^{185} \mathrm{Ir}$ have to be performed and will be pursued. For example, for $181 \mathrm{Ir}$ at $T=2.75 \mathrm{MeV}$ and $1=50$, $\mathrm{P}_{\mathrm{f}}=0.14$ compared to $\mathrm{P}_{f}=0.09$ for $185 \mathrm{I} r$. Adding the neutron emission due to the fast-fission evolution to the compound nucleus contribution (after proper weighting with the 1 distribution) the neutron multiplicitiy associated with fission before scission attains 3.3 compared to $3.6 \pm 0.6$ experimentally (see Table 3 ) $/ 20 \%$. At present, the asymmetric systems $20 \mathrm{Ne}+165 \mathrm{Ho}$ and $12 \mathrm{C}+175 \mathrm{Lu}$ (for $1>1 \mathrm{Bf}=0$ at $T=2.75 \mathrm{MeV}$ ) have an evolution within the fast-fission approach difficult to analyse. Immediately (or almost) in their time dependent behaviour, they reach an asymmetry beyond the Businaro-Gallone point and end up in fusion but still without fission barrier. Our model does not take into account, at present, the dynamical transition from large asymmetry configurations to the symmetric configuration (in $x$ space) leading to fission. This further investigation is underway. 


\section{$V$ - CONCLUDING REMARKS}

The theoretical dynamical approach with the treatment of particle evaporation provides a satisfactory explanation of particle emission associated to fission fragments. As soon as excitation energy is transmitted to the composite system, part of this energy is taken away by particle evaporation. On the whole history of the composite system, the time average excitation energy is less than the total available energy. This leads to qual ify these nuclei more warm than really hot.

We acknowledge fruitful discussions with many of our colleagues, especially $M$. Brack, J. Galin, D. Guerreau, C. Guet, D. Jacquet and G. Peaslee. We thank very much M. Marie for the careful typing of this manuscript.

\section{REFERENCES}

/1/ Delagrange, H. Ann. Phys. Fr. 3 (1982) 193

12/ Delagrange, H., Grégoire, C., Scheuter, F., Abe, Y., Z. Phys. A (1986), in press.

/3/ Bondorf, J.P., Donangelo, R., Mishustin, I.N., Pethick, C.J., Schulz, H. Sneppen, K., Nucl. Phys. A443 (1985) 321.

14/ Vinet, L., Sébille, F., Grégoire, C., Rémaud, B., Schuck, P., Preprint GANIL P.86-01, Phys. Lett. $B(1986)$ in press.

15/ Bonche, P., Lévit, S., Vautherin, D., Nucl. Phys. A427 (1984) 278.

16/ Grégoire, C., Ngô, C., Rémaud, B., Nucl. Phys. A383 (1982) 392.

17/ Bass, R., Nuclear Reactions with Heavy Ions, Springer-Verlag (1980).

18/ Grangé, P., Nucl. Phys. A428 (1984) 37c.

19/ Grangê, P., Li, Jung-Quing, , Weidenmueller, H.A., Phys. Rev. C27 (1983) 2063.

/10/ Lanza, E.G., Weidenmueller, H.A., Z. Phys. A323 (1986) 157.

/11/ Scheuter, F., Hofmann, H., Nuc1. Phys. A394 (1983) 477.

/12/ Kramers, H.A., Physica VII-4 (1940) 284.

/13/ Grégoire, C., Scheuter, F., Z. Phys. A303 (1981) 337.

/14/ Scheuter, F., Grëgoire, C., Hofmann, H., Nix, J.R., Phys. Lett. 149B (1984)

303.

115/ Bartel, J., Quentin, P., Phys. Lett. 152B (1985) 29.

16/ Brack, M., Guet, C., Hakansson, H.B., Phys. Rep. C123 (1985) 275.

/17/ Campi, X., Stringari, S., Z. Phys. A309 (1983) 239.

/18/ Ledergerber, T., Pauli, H.C., Nucl. Phys. A207 (1973) 1.

19/ Jacquet, D., Gali in, J., Borderie, B., Gardès, D., Guerreau, D., Lefort, M., Monnet, F., Rivet, M.F., Tarrago, X., Duek, E., Alexander, J.M., Phys. Rev. C32 (1985) 1594 .

/20/ Zank, W.P., Hilscher, D., Ingold, G., Jahnke, U., Lehmann, M., Rossner, H., Phys. Rev. C33 (1986) 519.

/21/ Ngô, C., Leray, S., Prog. Part. Nucl. 16 (1985).

122/ Jacquet, D., Duek, E., Alexander, J.M., Borderie, B., Galin, J., Gardès, D., Guerreau, D., Lefort, M., Monnet, F., Rivet, M.F., Tarrago, X., Phys. Rev. Lett. 53 (1984) 2226.

/23/ Töke, J., Swiatecki, W.J., Nucl. Phys. A372 (1981) 141. 